\title{
Efectividad de la citología cérvico-uterina para la detección temprana de cáncer de cuello uterino en el marco del sistema de salud de Colombia
}

\author{
Raúl Murillo', Ricardo Cendales², Carolina Wiesner ${ }^{3}$, Marion Piñeros ${ }^{4}$, Sandra Tovar ${ }^{3}$ \\ 1 Subdirección de Investigaciones, Instituto Nacional de Cancerología, Bogotá, D.C., Colombia \\ 2 Grupo de Radioterapia, Instituto Nacional de Cancerología, Bogotá, D.C., Colombia \\ 3 Grupo de Prevención, Instituto Nacional de Cancerología, Bogotá, D.C., Colombia \\ 4 Área de Salud Pública, Instituto Nacional de Cancerología, Bogotá, D.C., Colombia
}

Introducción. El cáncer de cuello uterino continúa siendo la primera causa de muerte por cáncer en mujeres colombianas, a pesar de la implementación desde 1991 de la detección temprana basada en la citología.

Objetivos. Evaluar la efectividad de la citología cérvico-uterina en Colombia.

Materiales y métodos. Se realizó un estudio de casos y controles (de población), equiparado por edad y entorno social. Los casos fueron mujeres de 25 a 69 años con cáncer invasor y los controles, mujeres sin cáncer invasor. Se trabajó en cuatro departamentos como escenarios diferenciales según el nivel de organización de la detección temprana y la mortalidad por esta causa. Los casos se seleccionaron aleatoriamente de los registros de patología en cada departamento (2005). Se aplicó una encuesta sobre los factores de riesgo y la historia de las citologías en los 72 meses previos.

Resultados. Se incluyeron 50 casos y 50 controles por departamento (400 sujetos). El promedio de edad fue de 48,4 años, el analfabetismo de 12,5\% y las personas sin aseguramiento de $13,8 \%$. El promedio de citologías fue más alto en los controles que en los casos $(p<0,01)$. El porcentaje de casos con antecedente de citología fue de 49,5\%. El uso de anticonceptivos y la falta de práctica de la citología estuvieron asociados con cáncer invasor (OR=2,53 y 3,54, respectivamente).

Conclusiones. La citología sigue siendo efectiva en la detección temprana del cáncer de cuello uterino. La efectividad está determinada más por la calidad de la prueba que por las coberturas de población. Es necesario revisar la normatividad del país. Los estudios de casos y controles son una herramienta útil en la evaluación de programas.

Palabras clave: neoplasias del cuello uterino, tamización masiva, evaluación de programas y proyectos de salud, citología, Colombia.

Effectiveness of cytology-based cervical cancer screening in the Colombian health system

Introduction. Despite the implementation of cytological screening since 1991, cervical cancer continuous to be the leading cause of cancer mortality among Colombian women.

Objectives. The effectiveness of cytology-based cervical cancer screening was subjected to review in the context of the Colombian health system.

Materials and methods. A case-control study was done. Invasive cervical cancer cases between 25-69 years were recruited and histopathological confirmation was required. Controls without invasive cancer were matched by age and neighborhood. Cases and controls were recruited in four Colombian provinces representing different settings for cervical cancer control with respect to program performance and mortality rates. The cases were randomly selected from the pathology in each province (year 2005). A survey of risk factors and cytology history in the previous 72 months was conducted.

Results. Fifty cases and 50 controls in each department were enrolled for a total of 400 subjects. The average age was 48.4 years, illiteracy $12.5 \%$, and persons without health insurance $13.8 \%$. 
The average number of Pap-smears was higher among controls $(p<0.01)$. Cases with a Papsmear in the previous 36 months was nearly half $(49.5 \%)$. Oral contraceptives and the lack of cytology were associated with invasive cervical cancer.

Conclusions. Cytology-based screening continued to be effective for early detection of cervical cancer in Colombia but its effectiveness was determined by quality of Pap-smears rather than by screening coverage. Governmental guidelines need to be revisited. Case-control studies provided a useful tool for evaluation of the screening program.

Key words: Uterine cervical neoplasms, mass screening, program evaluation, cytology, Colombia.

La tamización basada en citología cérvicouterina ha reducido la mortalidad por cáncer de cuello uterino en los países desarrollados pero, infortunadamente, este efecto no se ha logrado en la mayoría de los países en vías de desarrollo, incluida Colombia $(1,2)$. Las razones de la falta de efectividad en las naciones de bajos recursos se atribuyen, además de las limitaciones socioeconómicas, a problemas en la organización de los programas (3). Sin embargo, a la fecha, las evaluaciones de los programas se han centrado en la cobertura de la tamización, sin que exista relación clara entre ésta y el comportamiento de la mortalidad (4); por consiguiente, no existe suficiente información acerca de cuáles componentes de los programas son los responsables de la falta de efectividad.

En los años siguientes a la introducción de la tamización de cáncer de cuello uterino en América Latina, se realizaron análisis tendientes a valorar el impacto de la toma de citología sobre el riesgo de cáncer. Un estudio adelantado en Cali, Colombia, demostró que el riesgo de desarrollar cáncer invasor era 10 veces superior en las mujeres que no habían sido incluidas en la tamización que en las mujeres que sí habían sido incluidas $(R R=9,9)(5)$. En concordancia, el registro de cáncer de esa ciudad documentó, en los años precedentes al estudio, un incremento en el diagnóstico de cáncer in situ, frente a un

\footnotetext{
Correspondencia:

Raúl Murillo, Subdirección de Investigaciones, Instituto Nacional de Cancerología, Calle 1 No. 9-85, Bogotá, D.C., Colombia.

Telefax: (571) 3341360

rmurillo@cancer.gov.co

Recibido: 07/11/08; aceptado:05/03/09
}

descenso en el diagnóstico de cáncer invasor (5), lo cual evidencia un efecto positivo del programa de tamización con citología, similar a lo observado en países como Canadá (6).

Los datos confirmaron en su momento los beneficios de la citología en la reducción del riesgo de cáncer invasor; sin embargo, tal información no se relacionó con la mortalidad por cáncer de cuello uterino. Por otro lado, los programas iniciados en Colombia alrededor de los años setenta y ochenta, como el de Cali, fueron luego incorporados al programa nacional en 1990, pero, tras la reforma del sector salud en 1993, este programa perdió su carácter vertical y se transformó en un conjunto de actividades reglamentadas, pero desarrolladas por múltiples actores sin una unidad de coordinación (7).

Tras más de 15 años de la introducción del programa y de la reforma, la mortalidad por cáncer de cuello uterino en Colombia no muestra una tendencia clara al descenso y afecta, principalmente, a grupos de población con limitaciones para el acceso a los servicios y deficientes condiciones socioeconómicas $(8,9)$. La falta de efectividad de la tamización puede estar relacionada con diversos factores, que incluyen la cobertura de la población, la calidad en la toma y lectura de la citología, el acceso oportuno a la confirmación diagnóstica y el tratamiento, y la calidad del tratamiento, entre otros.

El realizar una evaluación integral de los distintos aspectos del programa es una tarea compleja que no puede adelantarse en un solo estudio. En el presente trabajo se analiza la diferencia en la historia de la citología en mujeres con cáncer invasor y los controles de población en cuatro departamentos de Colombia, con el 
fin de evaluar la efectividad de la citología en la detección temprana de la enfermedad como indicador de desempeño del programa y, a su vez, generar hipótesis documentadas sobre las causas de la falta de impacto del mismo en nuestro país.

\section{Materiales y métodos}

El estudio fue aprobado por el Comité de Ética e Investigaciones del Instituto Nacional de Cancerología de Colombia.

Se llevó a cabo un estudio de casos y controles en cuatro departamentos que representaban escenarios diferenciales según el nivel de organización de las actividades de detección temprana de cáncer de cuello uterino y según la mortalidad por esta causa. Dos departamentos tenían un adecuado desempeño en el número de citologías realizadas, pero presentaban tasas de mortalidad alta y baja, respectivamente (Caldas y Boyacá), y dos departamentos tenían un inadecuado desempeño en el número de citologías e, igualmente, tenían tasas de mortalidad diferenciales (Tolima y Magdalena) (10).

Se diseñó un estudio pareado asumiendo como casos las mujeres entre 25 y 69 años con diagnóstico histopatológico de cáncer invasor de cuello uterino y, como controles, las mujeres en el mismo rango de edad pero sin cáncer invasor (verificado por citología). Los controles se equipararon por edad al momento de la encuesta ( \pm 2 años) y por lugar de residencia (residentes en el mismo municipio y barrio del caso durante periodos similares). Se incluyeron mujeres que hubieran residido en los departamentos de estudio como mínimo por tres años y que no hubiesen estado embarazadas en ese lapso de tiempo. Se excluyeron los controles con antecedentes de histerectomía o de tratamientos relacionados con cáncer invasor o lesiones cervicales de cualquier grado y todas las mujeres cuya condición física o mental impedía el diligenciamiento adecuado del cuestionario. Los casos se seleccionaron aleatoriamente a partir del universo de pacientes con cáncer invasor identificado en los laboratorios de patología de cada departamento y diagnosticados durante el año 2005.
Enfermeras profesionales contactaron los casos y su respectivo control (ubicados en el mismo barrio y seleccionados aleatoriamente a partir de la información suministrada por vecinos del lugar, pero sin consultar los casos para su ubicación), y diligenciaron una encuesta estructurada sobre factores de riesgo para cáncer de cuello uterino y sobre la historia de citologías en los 48 meses previos al momento de la encuesta, incluidos los motivos de realización de las mismas. Los motivos de realización de las citologías fueron codificados y un médico epidemiólogo entrenado en cáncer y enmascarado con respecto a la condición de caso o control del sujeto, clasificó las citologías como diagnósticas o de tamización. Para los casos y los controles, las citologías motivadas en síntomas, las correspondientes a controles posteriores a una citología anormal (ASCUS o más) y las correspondientes a controles posteriores al tratamiento fueron excluidas del análisis. Para los casos, las citologías realizadas con posterioridad al diagnóstico fueron también excluidas.

\section{Análisis estadístico}

Se hizo doble digitación de la información en una base de datos validada. Se calculó un tamaño de muestra entre 40 y 50 pares para cada departamento basado en la prueba de McNemar, asumiendo un poder de $80 \%$, confianza $95 \%$, pruebas a una cola, razones de disparidad de 2 a 3 y una proporción de pares discordantes entre 0,2 y 0,3 .

El análisis se hizo con el programa SPSS $\AA$, versión 15.0. En el componente descriptivo se utilizaron medidas de tendencia central y de dispersión para las variables numéricas, y proporciones para las variables categóricas. La hipótesis nula de igualdad de proporciones se probó por la prueba de McNemar y la hipótesis nula de igualdad de medias por la t de Student para medidas repetidas si la variable numérica tenía distribución normal o, por la prueba de Wilcoxon, si no había distribución normal. Dada la suficiencia de la muestra los valores de $p$, finalmente, corresponden a pruebas de dos colas. En el análisis multivariable se utilizó un modelo jerárquico de regresión logística 
condicional seleccionando variables mediante el método paso a paso hacia atrás con criterio de permanencia de 0,05 . Se presentan análisis individuales a nivel departamental y un análisis para los cuatro departamentos.

\section{Resultados}

Se contactaron 428 mujeres (222 casos y 206 controles); 28 mujeres no fueron elegibles por embarazo en los tres años previos (10), negativa a conceder la entrevista (8), diagnóstico inadecuado (cáncer in situ, 6 casos, y cáncer invasor, un control) o residencia fuera del departamento (3). Se incluyeron, finalmente, 50 casos y 50 controles en cada departamento.

A 31 casos que desconocían el diagnóstico de cáncer se les dio información y se les orientó para el acceso al tratamiento. El promedio de edad fue de 48,4 años, la tasa de analfabetismo fue menor de $10 \%$, con excepción del departamento del Magdalena en donde fue de $26 \%$ (cuadro 1). La proporción de personas sin aseguramiento fue baja en los departamentos con buen desempeño en la realización de citologías y, mayor, en los departamentos restantes. Un porcentaje significativamente mayor de los casos no sabía leer ni escribir, inició relaciones sexuales y tuvo su primer parto antes de los 19 años, tuvo tres o más hijos, y tuvo mayor uso de anticonceptivos orales (cuadro 1).

El análisis por departamento mostró diferencias importantes con respecto al análisis global, por razones debidas a la disminución en el tamaño de la muestra; sin embargo, la práctica de, al menos, una citología de tamización en los últimos 36 meses fue una de las pocas variables que conservó diferencias estadísticamente significativas entre casos y controles (cuadro 2). En todos los departamentos, el promedio de citologías en los cinco años previos a la encuesta fue mayor en los controles que en los casos; Boyacá tuvo la menor diferencia entre casos y controles y, Magdalena, la más alta. A su vez, los departamentos con promedios de citologías más altos en el periodo evaluado fueron Tolima y Caldas. El porcentaje de casos con antecedentes de citología en los últimos tres años estuvo entre $38 \%$ y $56 \%$ (cuadro 2 ).
En el modelo multivariable sólo el tiempo de uso de anticonceptivos orales y la práctica de citología de tamización en los últimos 36 meses estuvieron significativamente asociados con el riesgo de cáncer en el análisis global. En el análisis por departamento, únicamente la práctica de citología en los últimos 36 meses mostró asociación significativa con OR bajo en Boyacá y Caldas (3,6 y 3,7, respectivamente), OR intermedio en Magdalena $(5,3)$ y un OR más cercano a la efectividad teórica en Tolima $(20,5)$ (cuadro 3).

\section{Discusión}

Los estudios de casos y controles se han utilizado para valorar la eficacia de la tamización basada en la citología en el diagnóstico de cáncer temprano $(5,11,12)$. A pesar de que esta eficacia ya está demostrada, el uso de tales estudios en la evaluación de un programa de salud pública ayuda a valorar las estrategias de tamización en las condiciones específicas de un sistema de salud y el aporte de distintos componentes en los resultados obtenidos. En este estudio, el alto porcentaje de ejecución de la muestra prevista y la equiparación de los casos y los controles por edad y por el entorno social, reducen la posibilidad de sesgos y dan mayor validez a los resultados obtenidos.

No obstante, nuestro estudio tiene varias limitaciones. La historia de citologías basada en encuestas introduce un sesgo de memoria que se ha propuesto como favorecedor de la tamización (13). En nuestro estudio, este sesgo se comporta en forma similar entre casos y controles, debido a la equiparación de las condiciones sociales mediante controles de población como se demuestra en el cálculo de los OR. La opción de revisar historias clínicas reduciría el sesgo de memoria, pero introduciría un sesgo de recolección de información ya que, ante la ausencia de programas estructurados en nuestro país, los datos sobre tamización se encontrarían más en los casos que en los controles, lo que los hace no comparables.

Una dificultad adicional es la clasificación de citologías con fines diagnósticos o de tamización. Los estudios previos eliminan siste- 
máticamente los registros en un periodo previo al diagnóstico de cáncer $(14,15)$. Nuestros cuestionarios incluyeron información detallada sobre los motivos de realización de la citología; consideramos que la evaluación ciega de un epidemiólogo asegura una valoración aceptable del tipo de citología, dadas las características del sistema de salud colombiano: no es frecuente en nuestro medio tener chequeos de rutina, máxime en una población como la de estudio, entre quienes el nivel educativo fue primaria en $48,5 \%$ y ninguno en $12,5 \%$.

Cuadro 1. Características de la población.

\begin{tabular}{|c|c|c|c|c|c|c|}
\hline Variable & Boyacá & Caldas & Magdalena & Tolima & Total & Valor de $p^{\star *}$ \\
\hline Edad (promedio) & 48,5 & 47,4 & 50,9 & 46,9 & 48,4 & 0,19 \\
\hline \multicolumn{7}{|l|}{ Estado civil } \\
\hline Soltera & 18,0 & 23,0 & 13,0 & 21,0 & 18,8 & \multirow[t]{3}{*}{0,42} \\
\hline Casada/unión libre & 63,0 & 55,0 & 73,0 & 65,0 & 64,0 & \\
\hline Separada/viuda & 19,0 & 22,0 & 14,0 & 14,0 & 17,3 & \\
\hline Analfabetismo & 10,0 & 5,0 & 26,0 & 9,0 & 12,5 & 0,01 \\
\hline \multicolumn{7}{|l|}{ Aseguramiento en salud* } \\
\hline Contribuyentes & 33,0 & 56,0 & 23,0 & 40,0 & 38,0 & \multirow[t]{3}{*}{ ND } \\
\hline Subsidiados & 63,0 & 41,0 & 57,0 & 28,0 & 47,3 & \\
\hline Sin seguro & 3,0 & 2,0 & 19,0 & 31,0 & 13,8 & \\
\hline \multicolumn{7}{|c|}{ Inicio de relaciones sexuales (años) } \\
\hline$<15$ & 13,0 & 12,0 & 15,0 & 14,0 & 13,5 & \multirow[t]{3}{*}{$<0,01$} \\
\hline 15 a 19 & 55,0 & 49,0 & 55,0 & 59,0 & 54,5 & \\
\hline$\geq 20$ & 32,0 & 39,0 & 30,0 & 27,0 & 32,0 & \\
\hline \multicolumn{7}{|l|}{ Edad al primer parto (años) } \\
\hline$<15$ & 1,0 & 0,0 & 3,0 & 3,0 & 1,8 & \multirow[t]{3}{*}{$<0,01$} \\
\hline 15 a 19 & 37,0 & 45,0 & 45,0 & 49,0 & 44,0 & \\
\hline$\geq 20$ & 62,0 & 55,0 & 52,0 & 48,0 & 54,3 & \\
\hline \multicolumn{7}{|c|}{ Número de hijos nacidos vivos } \\
\hline$\leq 2$ & 28,0 & 36,0 & 25,0 & 33,0 & 30,5 & \multirow[t]{2}{*}{$<0,01$} \\
\hline$\geq 3$ & 72,0 & 64,0 & 75,0 & 67,0 & 69,5 & \\
\hline \multirow{2}{*}{\multicolumn{7}{|c|}{$\begin{array}{l}\text { Más de tres compañeros sexuales } \\
\text { en la vida }\end{array}$}} \\
\hline & 39,0 & 36,0 & 16,0 & 24,0 & 28,8 & 0,01 \\
\hline $\begin{array}{l}\text { Uso de anticonceptivos } \\
\qquad 5\end{array}$ & & & & & & \multirow{3}{*}{0,05} \\
\hline$\leq 5$ & 86,0 & 69,0 & 82,0 & 70,0 & 76,8 & \\
\hline$\geq 5$ & 14,0 & 31,0 & 18,0 & 30,0 & 23,3 & \\
\hline \multirow{2}{*}{\multicolumn{7}{|c|}{ Fumadoras (más de 100 cigarrillos }} \\
\hline & 58,0 & 68,0 & 54,0 & 73,0 & 63,3 & $<0,01$ \\
\hline
\end{tabular}

* De las mujeres, $4 \%$ no reportan información sobre la afiliación al sistema de salud.

** El valor de $p$ representa las diferencias globales entre casos y controles (datos no mostrados) y se obtuvo mediante la prueba de McNemar para las variables categóricas y la prueba de Wilcoxon para las variables numéricas.

Los datos están expresados en porcentajes con excepción de la edad que se reporta como promedio.

Cuadro 2. Antecedentes en la práctica de citología.

\begin{tabular}{|c|c|c|c|c|c|c|c|c|c|c|c|}
\hline \multirow[t]{2}{*}{ Historia de citología } & \multicolumn{2}{|c|}{ Boyacá } & \multicolumn{2}{|c|}{ Caldas } & \multicolumn{2}{|c|}{ Magdalena } & \multicolumn{2}{|c|}{ Tolima } & \multicolumn{2}{|c|}{ Total } & \multirow{2}{*}{$\begin{array}{l}\text { Valor } \\
\text { de } p^{*}\end{array}$} \\
\hline & Caso & Control & Caso & Control & Caso & Control & Caso & Control & Caso & Control & \\
\hline $\begin{array}{l}\text { Antecedente de citología en } \\
\text { los últimos } 36 \text { meses (\%) }\end{array}$ & 48,0 & 68,0 & 56,0 & 80,0 & 38,0 & 70,0 & 56,0 & 90,0 & 49,5 & 77,0 & $<0,01$ \\
\hline $\begin{array}{l}\text { Promedio de citologías en } \\
\text { los últimos } 60 \text { meses }\end{array}$ & 1,12 & 1,57 & 1,39 & 2,0 & 0,6 & 1,44 & 1,37 & 2,9 & 1,11 & 1,97 & $<0,01$ \\
\hline
\end{tabular}

* Los valores de $p$ expresan las diferencias globales entre casos y controles y se obtuvo mediante la prueba de McNemar para las variables categóricas y la prueba de Wilcoxon para las variables numéricas. Los últimos 36 meses y los últimos 60 meses están en relación con el momento de la encuesta, tal como se describe en la sección de métodos. 
Cuadro 3. Análisis multivariable sobre riesgo de cáncer invasor del cuello uterino.

\begin{tabular}{|c|c|c|c|c|c|c|c|c|c|c|}
\hline \multirow[t]{2}{*}{ Variable } & \multicolumn{2}{|c|}{ Boyacá } & \multicolumn{2}{|c|}{ Caldas } & \multicolumn{2}{|c|}{ Magdalena } & \multicolumn{2}{|c|}{ Tolima } & \multicolumn{2}{|c|}{ Total } \\
\hline & OR & IC95\% & OR & IC95\% & OR & IC95\% & OR & IC95\% & OR & IC95\% \\
\hline \multicolumn{11}{|l|}{ Analfabetismo } \\
\hline Sí & 1 & & 1 & & 1 & & 1 & & 1 & \\
\hline No & 0,65 & $0,10-4,18$ & 6,31 & $0,43-91,81$ & 2,1 & $0,50-9,36$ & 0,2 & $0,01-2,58$ & 1,78 & $0,81-3,90$ \\
\hline \multicolumn{11}{|c|}{ Aseguramiento en salud* } \\
\hline Contribuyentes & 1 & & 1 & & 1 & & 1 & & 1 & \\
\hline Subsidiados & 1,26 & $0,20-7,99$ & ND & ND & 0,88 & $0,14-5,60$ & 7,36 & $0,86-63,25$ & 1,26 & $0,67-2,37$ \\
\hline Sin seguro & 2,66 & $0,10-74,76$ & ND & ND & 0,19 & $0,02-1,93$ & 2,42 & $0,52-11,32$ & 0,62 & $0,28-1,40$ \\
\hline \multicolumn{11}{|c|}{ Inicio de relaciones sexuales (años) } \\
\hline$\geq 20$ & 1 & & 1 & & 1 & & 1 & & 1 & \\
\hline 15 a 19 & 2,65 & $0,61-11,51$ & 2,19 & $0,34-14,19$ & 1,63 & $0,25-10,90$ & 1,78 & $0,26-12,36$ & 1,85 & $0,88-3,89$ \\
\hline$<15$ & 7,32 & $0,62-82,20$ & 1,06 & $0,14-7,69$ & 1,44 & $0,12-17,65$ & 4,23 & $0,17-103,15$ & 2,06 & $0,80-5,29$ \\
\hline \multicolumn{11}{|c|}{ Edad al primer parto (años) } \\
\hline$\geq 20$ & 1 & & 1 & & 1 & & 1 & & 1 & \\
\hline$<19$ & 2,72 & $0,79-9,36$ & 1,07 & $0,16-6,92$ & 1,92 & $0,24-15,22$ & 0,94 & $0,14-6,26$ & 1,25 & $0,66-2,37$ \\
\hline \multicolumn{11}{|c|}{ Número de hijos nacidos vivos } \\
\hline$\leq 2$ & 1 & & 1 & & 1 & & 1 & & 1 & \\
\hline$\geq 3$ & 1,00 & $0,33-3,02$ & 2,38 & $0,57-9,98$ & 3,25 & $0,65-16,20$ & 0,87 & $0,19-3,96$ & 1,34 & $0,77-2,34$ \\
\hline \multicolumn{11}{|c|}{$\begin{array}{l}\text { Más de tres compañeros sexuales } \\
\text { en la vida }\end{array}$} \\
\hline No & 1 & & 1 & & 1 & & 1 & & 1 & \\
\hline Sí & ,93 & $0,56-6,64$ & 0,54 & $0,12-2,42$ & 2,5 & $0,33-18,30$ & 2,59 & $0,73-9,24$ & 1,6 & $0,87-2,91$ \\
\hline \multicolumn{11}{|c|}{ Uso de anticonceptivos orales (años) } \\
\hline$\leq 5$ & 1 & & 1 & & 1 & & 1 & & 1 & \\
\hline$\geq 5$ & 1,48 & $0,30-7,32$ & 6,67 & $1,54-25,59$ & 7,63 & $0,41-140,80$ & 1,11 & $0,21-15,97$ & 2,53 & $1,32-4,86$ \\
\hline \multicolumn{11}{|c|}{ Citología en los 36 meses previos } \\
\hline Sí & 1 & & 1 & & 1 & & 1 & & 1 & \\
\hline No & 3,67 & $1,03-13,07$ & 3,76 & $0,96-14,72$ & 5,32 & $1,39-20,41$ & 20,57 & $2,52-168,20$ & 3,54 & $2,01-6,24$ \\
\hline
\end{tabular}

OR ajustados por las variables incluidas

Nuestros datos muestran, para todos los departamentos incluidos, que el antecedente de citología se relaciona con menor riesgo de cáncer invasor: en Caldas el OR no fue significativo, pero estuvo en el límite del intervalo de confianza. No obstante, la magnitud del efecto tuvo diferencias importantes entre grupos de población. Esta variación podría estar mediada en primera instancia por las coberturas de tamización de la población (diferencia en el antecedente de citología en los últimos tres años entre casos y controles). Concordantemente, losdepartamentos de Magdalena y Tolima mostraron los mayores gradientes de cobertura entre casos y controles (cuadro 2), lo cual se relaciona con la mayor efectividad observada en estos departamentos (OR de 5,32 y 20,57, respectivamente).

No obstante, la efectividad no mostró relación directa con la cobertura de la población en una perspectiva global, ya que, según la Encuesta
Nacional de Demografía y Salud de 2005, Caldas tiene las mayores coberturas, tanto para el antecedente de citología en los últimos tres años como para la medición a un año $(81,3 \%$ y $66,8 \%$, respectivamente), seguido en su orden, por Tolima (72,0\% y $44,3 \%)$, Boyacá $(70,7 \%$ y $43,3 \%$ ) y Magdalena (66,6\% y $47,6 \%$ ) ( $\left.{ }^{1} 6\right)$.

Un factor que pudiera relacionarse con la efectividad es la regularidad de los programas de tamización (frecuencia de la citología). Tolima y Caldas muestran un promedio mayor de citologías realizadas en los últimos cinco años $(2,1$ y 1,7 , respectivamente), lo cual podría indicar mayor regularidad en la asistencia a los programas. Sin embargo, las diferencias con los departamentos restantes no son significativas $y$, nuevamente, el gradiente entre casos y controles para esta variable es más alto en el Magdalena (cuadro 2), lo que coincide con la mayor efectividad en este departamento que en Caldas. 
Cabe destacar que, en general, hubo un alto porcentaje de casos de cáncer invasor con antecedentes de citología en los últimos tres años $(49,5 \%)$. Este dato indica que, quizá, la efectividad está mediada por factores diferentes a la cobertura e inherentes a la prueba de tamización, sin embargo, es de anotar que el estudio no indagó sobre el acceso a la confirmación diagnóstica (colposcopia-biopsia) tras el resultado de una citología positiva. En este sentido, el hallazgo de 31 casos que no conocían el diagnóstico puede estar relacionado con la falta de seguimiento de las citologías positivas.

Un componente esencial de la tamización basada en la citología cérvico-uterina es el control de calidad, el cual resulta de mayor relevancia por tratarse de una prueba dependiente del operador. A pesar de su importancia, pocos países en desarrollo evalúan de forma rutinaria la calidad de la citología y, entre quienes lo hacen, pocos utilizan la correlación citología-biopsia como indicador (4). En América Latina, únicamente Chile y Cuba tienen datos nacionales y sólo el primero utiliza el índice anotado previamente (4). Además, algunas experiencias adelantadas en la región demuestran que lograr niveles adecuados de calidad es una tarea difícil y que mantenerlos resulta aún más complicado $(17,18)$.

Al momento de esta publicación no se cuenta con datos sobre la calidad de la citología en los departamentos incluidos, ni en otras regiones del país. Sin embargo, la baja efectividad observada en Boyacá y Caldas, en donde la historia de citología no difiere mayormente entre casos y controles (cuadro 2), indican que el problema para los dos se centra probablemente en la calidad de la prueba, a diferencia de Magdalena y Tolima, en donde quizá pesa más la baja cobertura de la población de alto riesgo, hecho coincidente con una mayor tasa de analfabetismo y un promedio de citologías en los casos mucho menor.

En un estudio cualitativo de la percepción de los actores sobre el programa de tamización en los cuatro departamentos incluidos, se encontró que las actividades de control de calidad son prácticamente inexistentes (10), lo cual corroboraría la hipótesis planteada en torno a la calidad como el factor de mayor contribución en el hallazgo de una menor efectividad en Boyacá y Caldas. No obstante, algunos trabajos plantean la posibilidad de introducir un sesgo en contra de la citología si no se diferencian los casos detectados por tamización; esto puede ocurrir cuando los estudios se basan en datos rutinarios y se ha corregido asumiendo como caso únicamente los cánceres en estadio IB o más (14). Nuestro estudio incluye como controles casos de cáncer in situ lo que corrige parcialmente el problema; además, la limitación descrita no explicaría las diferencias observadas entre departamentos, toda vez que las historias de tamización de los casos son equiparables entre los que tuvieron baja efectividad y los que tuvieron efectividad media o alta (cuadro 2).

Apesar deque no es posible obtener conclusiones definitivas por la falta de información sobre la calidad de la prueba y el acceso a la confirmación diagnóstica, la información aportada por el estudio es muy relevante, debido a que evidencia que la problemática de la tamización de cáncer de cuello uterino, al menos para dos de los cuatro departamentos evaluados, no se centra en la cobertura sino en otros componentes del programa. Esto corrobora hallazgos previos que indican que aspectos distintos de la cobertura, como el seguimiento de las mujeres con citología positiva, podrían tener mayor impacto (19), y es contrario al énfasis puesto actualmente en la normatividad del país (metas de programación). Tal situación exige la obtención de información complementaria sobre otros componentes de los programas, la revisión urgente de la normatividad y el fortalecimiento integral de las estrategias de tamización.

\section{Conflicto de intereses}

Ninguno de los autores declara conflictos de interés para la publicación de este manuscrito.

\section{Financiación}

El trabajo fue realizado con recursos de inversión de la Nación suministrados al Instituto Nacional de Cancerología y designados bajo el código institucional 41030311-05. 


\section{Referencias}

1. Kitchener H, Castle P, Cox J. Achievements and limitation of cervical cytology screening. Vaccine. 2006;24:63-70.

2. Ferlay J, Bray F, Pisani P, Parkin DM. Cancer incidence, mortality and prevalence worldwide. Lyon: IARC Press; 2002.

3. Sankaranarayanan R, Madhukar-Budukh A, Rajkumar R. Effective screening programmes for cervical cancer in low- and middle-income developing countries. Bull World Health Organ. 2001;79:954-62.

4. Murillo R, Almonte M, Pereira A, Ferrer E, Gamboa O, Jerónimo J, et al. Cervical cancer screening programs in Latin America and the Caribbean. Vaccine. 2008;26:L3748.

5. Aristizábal N, Cuello C, Correa P, Collazos T, Haenszel W. The impact of vaginal cytology on cervical cancer risks in Cali, Colombia. Int J Cancer. 1984;34:5-9.

6. Liu S, Semenciw R, Probert A, Mao Y. Cervical cancer in Canada: changing patterns in incidence and mortality. Int J Gynecol Cancer. 2001;11:24-31.

7. Murillo R. Control del cáncer de cuello uterino en Colombia: triunfos y desafíos de la tamización basada en la citología cérvico-uterina. Biomédica. 2008;28:46870.

8. Piñeros $\mathbf{M}$, Hernández $\mathbf{G}$, Bray $\mathbf{F}$. Increasing mortality rates of common malignancies in Colombia: an emerging problem. Cancer. 2004;101:2285-92.

9. Murillo R, Piñeros M, Hernández G. Atlas de mortalidad por cáncer en Colombia. Bogotá: Instituto Nacional de Cancerología, Instituto Geográfico Agustín Codazzi; 2004.

10. Wiesner C, Murillo R, Piñeros M, Tovar S, Cendales R, Gutiérrez MC. El control del cáncer cervicouterino en Colombia: percepción de los actores del sistema de salud. Rev Panam Salud Pública. 2008; en imprenta.
11. Herrero R, Brinton LA, Reeves WC, Brenes MM, de Britton RC, Gaitan E, et al. Screening for cervical cancer in Latin America: a case-control study. Int J Epidemiol. 1992;21:1050-6.

12. Hernández-Ávila M, Lazcano-Ponce EC, de Ruiz PA, Romieu I. Evaluation of the cervical cancer screening programme in Mexico: a population-based case-control study. Int J Epidemiol. 1998;27:370-6.

13. Zappa M, Visioli CB, Ciatto S, lossa A, Paci E, Sasieni P. Lower protection of cytological screening for adenocarcinomas and shorter protection for younger women: the results of a case-control study in Florence. BJC. 2004;90:1784-6.

14. Sasieni P, Adams J, Cuzick J. Benefit of cervical screening at different ages: evidence from the UK audit. Of screening histories. BJC. 2003;89:88-93.

15. Andrae B, Kemetli L, Sparén P, Silfverdal L, Strander B, Ryd W, et al. Screening-preventable cervical cancer risks: evidence from a nationwide audit in Sweden. J Natl Cancer Inst. 2008;100:622-9.

16. Piñeros M, Cendales R, Murillo R, Wiesner C, Tovar S. Cobertura de la citología de cuello uterino y factores relacionados en Colombia, 2005. Rev Salud Pública (Bogotá). 2007;9:327-41.

17. Lazcano-Ponce EC, Moss S, Alonso P, Salmerón J, Hernández $\mathbf{M}$. Cervical cancer screening in developing countries: why is it ineffective? The case of Mexico. Arch Med Res. 1999;30:240-50.

18. Lewis MJ. A situational analysis of cervical cancer in Latin America and the Caribbean. Washington D.C.: Pan American Health Organization; 2004.

19. Gamboa OA, Chicaíza L, García-Molina M, Díaz J, González M, Murillo R, et al. Cost-effectiveness of conventional cytology and HPV-DNA testing for cervical-cancer screening in Colombia. Salud Pub Mex. 2008;50:276-85. 